Paper to be presented at the ANS 1990 Fast Reactor Safety Meeting, Snowbird, Utah, Aligust 12-16, 1990.

CONF-900304--22

DE90 011158

\title{
A MEASUREMENT-BASED METHOD FOR PRFDICTING MARGINS AND UNCERTAINTIES FOR LINPROTECTED ACCIDENTS IN THE INTEGRAL FAST REACTOR CONCEPT
}

by

\author{
R. B. ViTim \\ Argonne National Laboratory \\ 9700 S. Cass Avenue \\ Argonne, IL 60439
}

\begin{abstract}
DISCLAIMER
This report was prepared as an account of work sponsored by an agency of the United States Government. Neither the United States Government nor any agency ther eof, nor any of their employees, makes any warranty, express or implied, or assumes any legal iability or responsibility for the accuracy, completeness, or usefulness of any information, apparatus, product, or process disclosed, or represents that its use would not infringe privately owned rights. Reference herein to any specific coinmercial product, process, or service by trade name, trademark, manufacturer, or otherwise does not necessarily constitute or imply its endorsement, recommendation, or favoring by the United States Government or any agency thereuf. The views and opinions of authors expressed herein do not necessarily state or reflect those of the United States Government or any agency thereof.
\end{abstract}

\footnotetext{
*Work supported by the U.S. Department of Energy, Nuclear Energy Programs under contract W-31-109-ENG-38.
} 


\title{
A MEASUREYENT-BASED METHOD FOR. PREDICTING \\ MARGINS AND UNCERTAINTIES FOR UNPROTECTED ACCIDENTS \\ IN THE INTEGRAL FAST REACTOR CONCEPT
}

\author{
R. B. Vilim \\ Argonne Nationa1 Laboratory \\ Argonne, Illinois 60439 , U.S.A.
}

ABSTRACT

A measurement-based method for predicting the response of an LMR core to unprotected accidents has been developed. The method processes plant measurements taken at normal operation to generate a stochastic model for the core dynamics. This model can be used to predict three sigma confidence intervals for the core temperature and power response. Preliminary numerical simulations performed for EBR-II appear promising.

\section{INTRODUCTION}

If the economic and safety benefits of an inherent shutdown capability in the Integral Fast Reactor (IFR) concept are to be realized, then the concept of passive safety, and the associated risk reduction, must be incorporated in the plant probabilistic risk assessment. In order to claim passive safety, however, it must be shown that sufficient inherent feedback mecinanisms for safe shutdown are present in the plant as built. The only way to demonstrate this is to routinely perform dynamic tests on the plant. An analogous situation exists in light water plants. Tests are periodically performed to verify that the Engineered Safety Systems mee: design requirements. These tests are mandated by the Technical Specifications upon which the reactor has been licensed.

The goal is to show that the required shutdown cavability is in place. To do so, periodic and unobtrusive testing of plant dynamics will be performed. The plant measurements from these tests will be processed to yield a dynamic model that comprises a measured basis upon which the response to unprotected accidents will be predicted. If the response is within safety limits, then it. will be concluded that the mechanisms for safe shutdown are in place.

Other approaches exist for predicting the response of the core 1.2 but they do not make use of current mexumrements and as a result suffer two shortcomings. First, estimates fur uncertainty magnitudes made without consideration for current operating data will always be larger than those obtained by direct measurement. Since the core rating must be decreased as 
the uncertainty increases, an economic penalty is imposed. In principle, the minimum uncertainty levels for the method proposed in this paper depend only on measurement noise levels. Second, a direct confirmation of model validity requires that the identified model be compared against current measurements and that the goodness of the fit be consistent with the statistical properties of the identified model. This comparison can be done only if current measurements are available.

While the preferred form of the identified model is one developed from measured data without regard to model structure, some structure beyond that of a transfer function is required to handle nonlinearities. A linear model simply cannot represent core behavior during a rapid loss of flow.

In addition to the requirement for a measurement-ba:ied approans and the need for some nonlinear model structure, there are other facto:s that influence the type of scheme used to generate the model. First, experience at EBR-II ${ }^{3,4}$ indicates that neither process noise in the core nor ras drop tests provide sufficient excitation energy to identify dynamic beh; ior. Test signals in the form of perturbations to reactor flowrate, rod fosition and inlet temperature will have to be introduced. Second, to be practical from an operations standpoint, this excitation must take place at normal power. Third, measurement noise and its effect on the uncertainty of the model prediction must be explicitly dealt with. Finally, a statistical test is required to verify that the identified model of the core dynamics is consistent with the measurements.

This paper describes a methodology developed to meet these goals. The methodology is a synthesis of existing methods and an extension of cthers.

\section{MODEL EQUATIONS}

The system to be identified is in general composed of three elements: sensors, actuators and process hardware. The behavior of each element is governed by the conservation laws, and these laws are normally represented in lumped parameter form. The system equations then take the form

$$
\frac{d}{d t} x(t)-f_{c}[x(t) \cdot \mu(t)]
$$

and

$$
y(t)-g[x(t), u(t)]
$$

where

$$
\begin{aligned}
& \underline{x}(t)=\mathrm{n} \times 1 \text { state vector } \\
& \underline{u}(t)=\mathrm{m} \times 1 \text { input vector } \\
& \underline{y}(t)=\mathrm{r} \times 1 \text { output vector. }
\end{aligned}
$$


The stochasiic or randorn components of the plant behavior are assumed superimposed on the deterministic model of Eq. (1). This equation is transformed to discrete-time form and augmented with the random vectors $\xi_{u}(k), \xi_{x}(k)$ and $n(k)$ to give

$$
\ddot{u}(k+1)-f_{d}\left[x(k), \underline{u}(k), \xi_{u}(k)\right]+\xi_{x}(k)
$$

and

$$
\underline{y}(k)-\underline{g}\left[x(k), \underline{u}(k), \xi_{u}(k)\right]+\eta(k) .
$$

where $k$ denotes the sample time.

\section{METHODOLOGY}

The methodology is sufficiently general that it can be used to determine a probabilistic model for any system whose underlying basis is a set of ordinary differential equations.

A prudent first step is to determine the minimum model order necessary to describe the plant. The model order that best describes the measured data is determined using Akaike's minimum information criterion (AIC). ${ }^{5}$ The criterion is used as follows. For order one we identify at the full power operating point, a discrete-time linear canonical stochastic model. The maximum of the likelihood function is a by-product and we use this to calculate AIC. This procedure is repeated for orders $2,3, \ldots$ until the minimum AIC is found. The model associated with the minimum AIC is, according to Akaike, the one that best describes the data at the operating point.

The model order, and time constants which are output in the above step, and a knowledge of the underlying physics are used to hypothesize a model structure whose parameter values are to be estimated. The only constraint on the form of the structure is that it can be written in the form of Eq. (2). In addition to $\underline{u}(k)$ representing a forcing function, it can also be used to represent a model parameter. For example, if the ith element of $\underline{u}(k)$ is to represent the deterministic parameter $\alpha_{d}$, then $\underline{u}(k)_{i}$ $=\alpha_{d}$ and $\operatorname{Var}\left[\xi_{u}(k)_{i}\right]=0$ in Eq. (2). Alternatively, if the ith element of $\underline{u}(k)$ is to represent the stochastic parameter $\alpha_{s}$, then $\underline{u}(k)_{i}-E\left[\alpha_{s}\right]$ and $\operatorname{Var}\left[\xi_{u}(k)_{i}\right]=\operatorname{Var}\left[\alpha_{s}\right]$. The symbols $E$ and $\operatorname{Var}$ denote expected value and variance, respectively.

The parameter values sought are those that maximize the logarithm of the likelihood function of Eq. (2),

$$
\max _{\underline{\alpha}} \operatorname{In} f\left[\underline{\mathrm{V}}_{\mathbf{m}}(1), \ldots, \underline{I}_{\mathrm{m}}(\mathrm{n}) \mid \underline{\alpha}\right]
$$

where the dependence on the parameters $\underline{\alpha}$ has been written explicitly. 
If the plant output measurements iIs $\underline{y}_{n}(1), \ldots, \underline{y}_{m}(n)$, then

$$
\begin{aligned}
& \ln f\left[y_{m}(1), \ldots, y_{m}(n)\right] \\
& --\frac{1}{2} \sum_{k=1}^{n}\left[I_{m}(k)^{T} \sum(k \mid k-1)^{-1} I_{m}(k)+x \ln 2 \pi+\ln \left|\operatorname{det} \sum(k \mid k-1)\right|\right]
\end{aligned}
$$

where

$$
I_{m}(k)-\underline{y}_{m}(k)-g[\hat{x}(k \mid k-1), \underline{u}(k), 0]
$$

and where $\Sigma(k \mid k-1)$ is the covariance matrix of the measurement residual and $\boldsymbol{z}(k \mid k-1)$ is the Kalman filter state estimate.

As a necessary condition for the model to be declared valid, we require that the statistical properties of the identified model be consistent with the measured data. A reasonable test is to check to see that the measurement residuals are white as they should be. This can be done by computing the covariance of measurement residuals displaced in time and looking to see that

$$
E\left[I(k) I(k+1)^{r}\right]=0,1 \neq 0 \text {. }
$$

The test for measurement residual whiteness used in this paper is that of Peterson. ${ }^{6}$ He proposes the matrix

$$
R(1)=\frac{1}{N-1} \sum_{k=1}^{N-1} \tilde{I}(k) \tilde{I}(k+1)^{T}
$$

where

$$
\tilde{I}(k)-\sum(k \mid k-1)^{-1 / 2} I(k)
$$

as a measurement of the covariance between residuals. The statistical properties of $R(1)$ vere, however, not given by him so they were derived independently as part of this work.

The validity test is as follows. The values of the elements of $R(I)$ are computed from plant measurements $I_{m}(1), \ldots, Y_{n}(N)$ using Eq. (6) to give

$$
\begin{aligned}
& R(1)_{i j} *= \text { value of } R(1)_{i j} \text { computed using plant measurements } \\
& y_{\pi}(1), \ldots, Y_{\pi}(N) .
\end{aligned}
$$


Now if Eq. (2) is a valid representation of the plant and if the errors introduced by the linearization are small, then $R(1)_{i j}^{*}$ must be consistent with the statistical properties of $R(1)_{1 j}$. To be specific, if the identified model given by $\mathrm{Eq}$. (2) is a valid representation of the measurements $\mathrm{V}_{\pi}(1), \ldots, Y_{n}(N)$, then for $f$ defined to be the fraction of $R(I) *$ elements for which

$$
\frac{\left|R(1)_{i j}-\mu_{R(1)_{i j}}\right|}{\sigma_{R(1)_{i j}}}<3
$$

we must have $f>0.995$ where $\mu$ denotes the mean and $\sigma$ the standard deviation.

The identified model can be used to predict the uncertainty in the plant response for a given input forcing function. The state variables $\underline{x}(k)$ and the outputs $y(k)$ in $E_{q}$. (2) correspond to temperatures and powers that are of interest from a safety standpoint. Further, these quantities are random variables whose statistics can be calculated. However, in Eq. (2), $\underline{\mathbf{v}}(k)$ is a sensor signal which includes the effect of sensor noise. We are more interested in predicting the process variable itself, not the noise corrupted version output by the sensor. The more appropriate form of Eq. (2) for prediction is

$$
x(k+1)-f_{d}\left[x(k), \underline{u}(k), \xi_{u}(k)\right]+\xi_{x}(k)
$$

and

$$
z(k)-g\left[x(k), \underline{u}(k), \xi_{u}(k)\right]
$$

where the output measurement noise term, $\underline{\underline{ }(k)}$, has been dropped.

\section{APPLICATION}

The methodology was evaluated by processing simulated measurements generater for the EBR-II reactor. This approach provides a starting point for the eventual demonstration of the methodology at the EBR-II plant using real data.

The results presented here considered only the effect of primary flowrate on reactor behavior. The effect of external reactivity and inlet temperature are to be studied in future work.

The simulation model used in this example while relatively simple -. it consists of the point kinetics equation with one precursor and the prompt jump approximation and a lumped parameter equation for the control rod -- predicts the core power behavior observed in EBR-II flow coastdown tests quite well. The solution for quasi-static changes in flow depends on a parameter $\alpha_{\mathrm{qs}}$, a function of all reactivity feedbacks. The solution for more rapid changes depends on the additional parameters $\alpha_{d 1}$, the one-group 
neutron precursor half-life, $\alpha_{\mathrm{d} 2}$, except the control rods, and $\alpha_{\mathrm{i} 3}$, constant. a function of all reactivity feedbacks the control rod temperature time

Measurement data were generated by adding white noise to reactor power and primary flow obtained from a noise-free simulation.

\section{Quasi-Static Identification}

A slowly varying transient in which some parameters do not affect the quasi-static plant response presents an opportunity to identify a subset of parameters. In turn, the task of identifying the remaining parameters through a fast transient is made easier. Specifically, if flow is varied in a quasi-static manner, then the parameters $\alpha_{d 1}, \alpha_{d 2}$, and $\alpha_{d 3}$ do not affect the power. This leaves the expected value of $\alpha_{q s}$, the variance of $\alpha_{\bar{q} s}$, the variance of the dieasured flowrate, $\xi_{\overline{\mathrm{N}}}(k)$, and the variance of the measured power, $\eta_{\bar{j}}(k)$, to be identified. The state equation errors $\xi_{\overline{0}}(k)$ and $\xi_{\tau_{a}}(k)$ are set to zero for the present.

A quasi-static transient was initiated by ramping flowrate from 100 to 85 percent and back again over a period of one hour. Simulated measuremerts were generated by adding white noise to the flowrate forcing function and to the calculated reactor power.

The parameter values identified from the quasi-static transient simulated measurements are shown in Table $I$. As seen in the table, the "initial" values used to start the parameter search algorithm are a factor of two different from the "actual" values used to generate the simulated measurements.

\section{Model Order Identification}

A determination of model order was made by perturbing the plant at full power and processing the measurements using Akaike's criterion. The input signal consisted of a pseudo-random binary sequence superimposed on the full power primary flowrate. The AIC criterion is plotted as a function of model order in Fig. 2. The results indicate that flow perturbations of at least five percent but no more than ten percent are needed to correctly determine the model order. Further, the system is very nearly first order which is consistent with the fact that the control rod feedback coefficient is small.

Based on the above findings, flowrate perturbations of five percent and a system order of one were determined as appropriate for performing dynamic identification. One concludes that the effect of the control rod on plant dynamic behaviour is minor.

\section{Dynamic Identification}

Identification was performed for the first order system obtained by setting the control rod driveline time constant to zero. In doing so we find the parameters $\alpha_{\mathrm{d} 2}$ and $\alpha_{\mathrm{d} 3}$ assume fixed values in the problem. 
We are left with a reduced set of parameters to be estimated and these are listed in Table II.

This first order system was identified using simulated measurement data generated from the second order model. The input signal consisted of the signal in the quasi-static case with a plus and minus five percent pseudo-random binary signal superimposed on this.

The parameter values identified are shown in Table II.

Test for Model Validity

The validity test given by Eq. (8) was applied to the identified model. The model passed the test.

\section{Prediction Uncertainty}

The validated model was used to predict the response of the core to an unprotected loss of flow accident. A hyperbolic flow decay with a halving time of five seconds was used and is typical of a flow coastdown in EBR-II following a loss of electric power to the primary pumps.

The resulis of the uncertainty prediction are shown in Fig. 2. The solid line is the power-to-flow ratio given by the model used to generate simulated measurements throughout this work. The two dotted lines show the three-sigma confidence level of the identified model. As it should, the identified model prediction envelopes the response of the model that was used to generate the simulated measurements.

\section{SUMLLARY AND FUTURE WORK}

A method was developed to obtain a probabilistic model for the dynamic behavior of a reactor core from measurements made at normal operation. The method tests initially for the dynamic order of the plant. Time constants are then determined once the order is known. From a knowledge of time constants and the underlying physics, one develops a stochastic model structure to describe the plant. The parameter values in this structure are then selected to maximize the likelihood that the model produced the measured data. A validity test is then used to check for statistical consistency between model and measuremenis. The model once validated can be used to predict three-sigma core response uncertainty levels for different forcing functions.

It. is envisioned that this capability will be used to predict the response of an as-built IFR core to unprotected accidents. This capability will be required if credit for a passive shutdown $c$ pability is to be taken in the plant probabilistic risk assessment. Measurements will be gathered in an unobtrusive manner and on a periodic basis. The resulting model will be driven by core boundary conditions - primary flowrate, reactor inlet temperature and external reactivity - corresponding to five classes of 
unprotected accidents: loss of flow, loss of heat sink, rod withdrawal, primary pump overspeed and chilled core inlet temperature. The three-sigma confidence levels generated by the model will then be compared against cladding eutectic, sodium boiling and fuel melting safety limits. If the predicted response is within safety limits, then it will be concluded that the mechanisms for safe shutdown are in place.

The method was evaluated by processing simulated measurements from the EBR-II reactor. Although the simulation model is relatively simple, it predicts the core behavior observed in EBR-II tests quite well. The method appears very promising. The model order test proved effective for determining the minimum signal-to-noise ratio required, for determining the actual system order and for determining an appropriate model order. In particular, pseudo-random binary signals of ten percent peak-to-peak superimposed on full power flowrate proved sufficient for identifying core dynamics.

Future work will be directed toward eventual demonstration in EBR-II. The next step will be to generate new simulated measurements using the more detailed modeis in the SASSYS code. A more detailed investigation of the effect of the input signal's energy spectrum and time duration on the uncertainty in the identified model will be made. In addition to including primary flowrate as a forcing function as was done in this report, inlet temperature and external reactivity will also be examined.

\section{REFERENCES}

1. R. S. MAY, J. M. SORENSON and R. E. ENGEL, "Probabilistic Methods for Evaluating Operational Transient Margins and Uncertainties, " Nuclear Science and Engineering, 103, $81-93$ (1989).

2. C. J. MUELLER and D. C. WADE, "Probabilities of Successful Inherent Shutdown of Unprotected Transients in Innovative Liquid Metal Reactors," Proc. Int. Todical Meeting Safety of Next Generation Power Reactors, Seattle, Washington, May 1-5, 1988, p. 704, American Nuclear Society (1988).

3. D. D. EBERT, "An Inherent Noise Analysis Investigation on the Experimental Breeder Reactor II, "Nuclear Science and Engineering, 55, 470 (1974).

4. A. J. FOLTMAN, "Limitations of EBR-II Rod-Drop Tests and Proposed Improvements Using Frequency-Response Testing," Proceedings Second Power Plant Dynamics, Control, Testing Symposium, Knoxville, Tennessee (1975).

5. H. AKAIKE, "A New Look at The Statistical Model Identification," IEEE Transactions on Automatic Control, Vol. AC-19, No. 6 (1974).

6. D. W. PETERSON, "Residual Processes for Model Validation and Bad-Data Detection," unpublished data, Massachusetts Institute of Tech. (1976). 


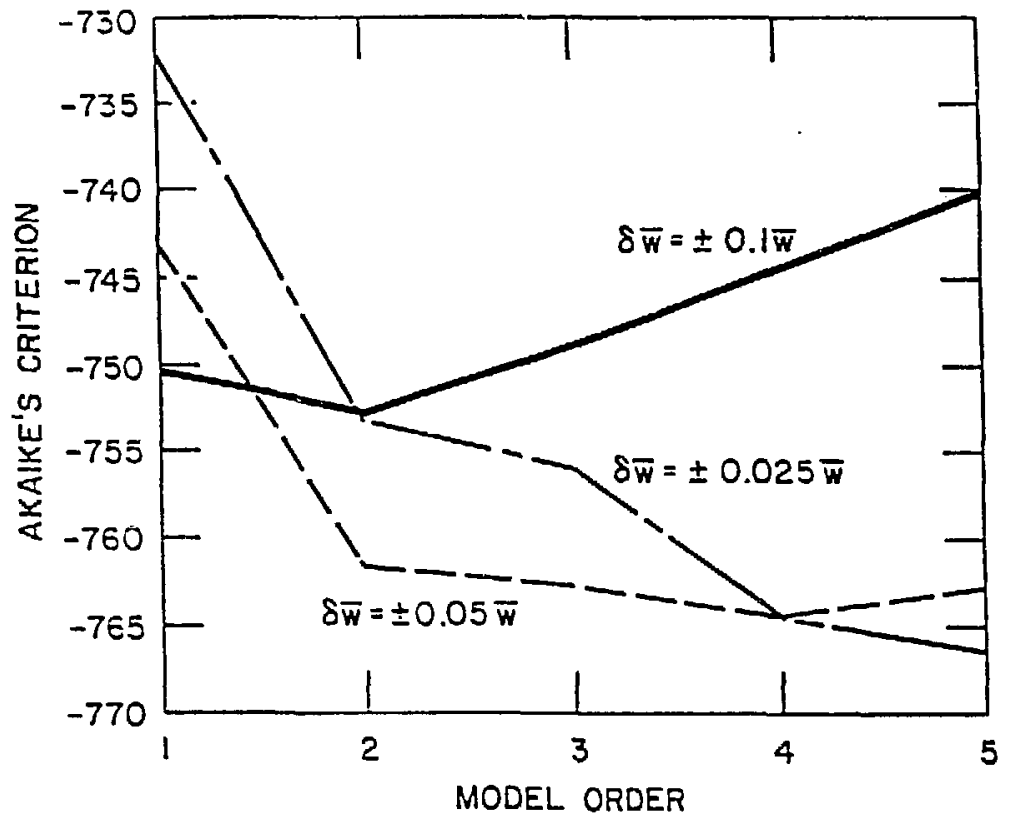

Fig. 1. Akaike's Criterion as a Function of Primary Flowrate Perturbation Magnitude.

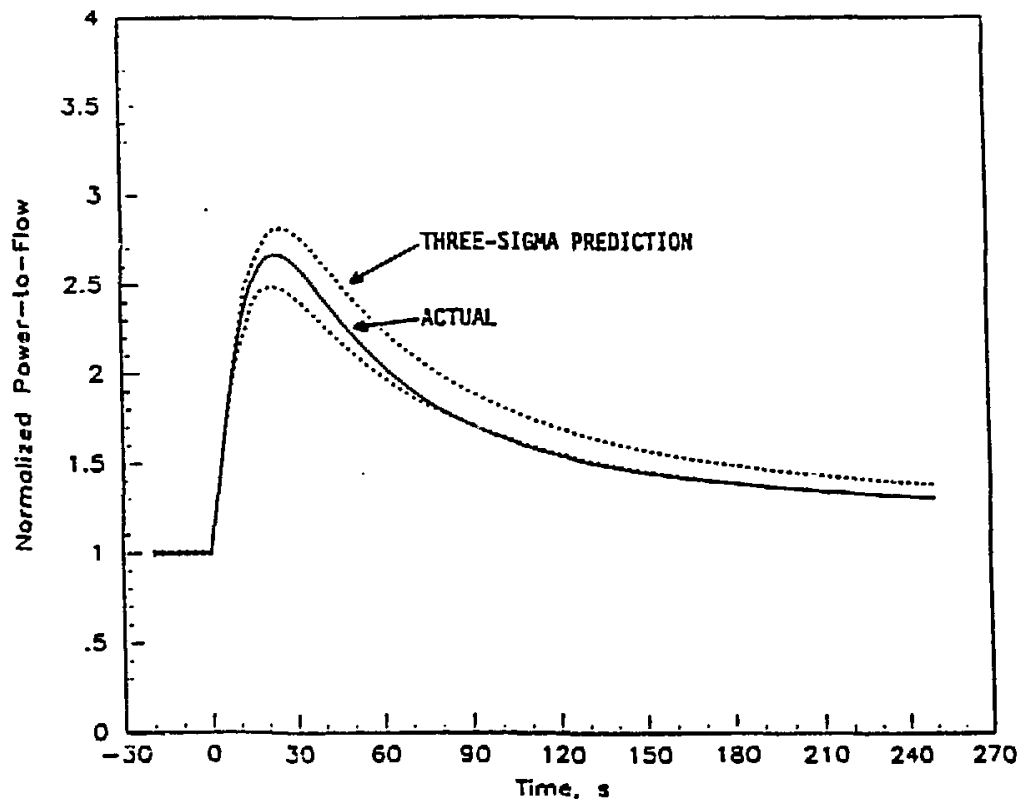

Fig. 2. Three-Sigma Power-to-Flow Uncertainty Prediction for Flow Coastdown. 